\title{
Performance Analysis of ANN based Gait Recognition
}

\author{
Taniya Sharma"1 Satnam Singh Dub², Bhanu Gupta ${ }^{3}$ \\ ${ }^{* 1}$ Department of ECE, SSCET, IKGPTU, Pathankot, Punjab, India \\ ${ }^{2}$ Department of ECE, SSCET, IKGPTU, Pathankot, Punjab, India \\ ${ }^{3}$ Department of AE\&IE, M.B.S.C.E.T, University of Jammu, Jammu, J\&K, India
}

\section{ABSTRACT}

It is well-known that biometrics are a powerful tool for reliable automated person identification. Automatic gait recognition is one of the newest of the emergent biometrics and has many advantages over other biometrics. The most notable advantage is that it does not require contact with the subjects nor does it require the subject to be near a camera. This work employs a gait recognition process with binary silhouette-based input images and Artificial Neural Network (ANN) based classification in MATLAB. The performance of the recognition method depends significantly on the quality of the extracted binary silhouettes. In this work, a computationally low-cost fuzzy correlogram based method is employed for background subtraction. Even highly robust background subtraction and shadow elimination algorithms produce erroneous outputs at times with missing body portions, which consequently affect the recognition performance. Frame Difference Energy Image (FDEI) reconstruction is performed to alleviate the detrimental effect of improperly extracted silhouettes and to make the recognition method robust to partial incompleteness. Subsequently, features are extracted via two methods and fed to the BPNN (Back Propagation Neural Network based classifier which uses feature vector (exemplars) to compute similarity scores and carry out identification using weight vectors i.e. Frame-toExemplar-Distance (FED) vector. The FED uses the distance measure between pre-determined feature vectors and the weight vectors of the current frame as an identification criterion. The ANN performance is evaluated for recognition and speed parameters at different training gait angles.

Keywords : Gait, Biometrics, ANN, FDEI, FED, MATLAB

\section{INTRODUCTION}

This Gait is the way locomotion is achieved using human limbs. Differences in limb movement patterns, velocity, forces, kinetic energy cycles and changes in the contact with surface characterizes different gaits. The methods and mechanics of human ambulation the bipedal gait - are quite unique in the nature. In relation to human walking, gait refers to the style or manner of walking, rather than the walking process itself [1]. Once gait is learned it becomes almost subconscious. Individual gait is dependent on many variables such as surface properties, confidence, mood, quantity and quality of sensory information (e.g. light levels, noise, and distractions), any chemical effects on the body (such as alcohol or medication), energy levels, mental alertness and muscle fatigue.

Walking mainly consists of the ability to maintain the equilibrium and the ability to initiate and maintain a rhythmic stepping motion - locomotion. In conjunction with these two abilities, there are other many contributing factors involved in walking such as the skeletal system with the joints as well as neuro-muscular system. 
The performance of any recognition system is dependent on the quality of gait data. The use of advanced computer vision processing algorithms has yielded some improvement, but this is often at the expense of computational complexity of gait databases with the aim to progress from laboratory based studies on small population [2].

Gait recognition aims essentially to address this problem by recognizing people based on the way they walk. The study of human gait has been increased extensive interests in various fields such as clinical analysis, computer animation, athletic performance analysis, visual surveillance, robotics and biometrics. Gait Biometrics is a new powerful tool for reliable human identification and it makes use of human physiology characteristics such as face, iris, finger prints and hand geometry for identification.

The systematic study of human locomotion or animal movement by using the eye and brain of the observers, in combination with instrumentation for measuring the body movements, activities of the muscles or body mechanics is known as Gait Analysis. The walking movement that is achieved by the motion of the human limbs is known as human gait. The human gait is defined as bipedal (i.e. walking on 2 feet) and biphasic which is the result of sinuous movements along with forward progression of the different body parts [3].

The gait is characterized by kinematics. Static and dynamic features such as speed, stride length, cadence rate, and angular and linear displacements of body segments can be extracted by bio-mechanical analysis of walking human body. Psychophysical studies showed that people can recognize/identify friends, and gender from the way they walk. Gait as a biometrics feature has gained increasing attention, as it can be collected from a distance using a video camera. In controlled environments like work places, banks and airports it is preferred to use non-intrusive authentication methods.

Gait recognition can be broadly classified into two types: model-based and model-free approach. Model based approaches purpose to explicitly model human body or motion and performs model matching in each frame of a walking sequence. Model based methods aim to model human body analysis of the parts of body such as hand, torso, thigh, legs and foot to perform model matching in each frame of a walking sequence to measure these parameters. The study of human gait has been increased extensive interests in various fields such as clinical analysis, computer animation, athletic performance analysis, visual surveillance, robotics, and biometrics. Moreover, human motion analysis has many challenging issues, because the highly flexible structure and self-occlusion of the human body mandates complicated processes for the segmentation and analysis of motion. As the human gait is known to be one of the most universal and complex of all human activities, it has been studied in medical science, biomechanics and psychology for decades. In computer vision, automated person identification by gait has been investigated. The human gait is usually described by kinetic or kinematic characteristics, and the pattern of rotation angles around the joints has been modelled as a pendulum, the motion of which is characterized by simple harmonic motion. In addition, each person appears to have his or her own characteristic of gait pattern. There is much evidence from psychophysical experiments and gait analysis that gait patterns have characteristics of uniqueness for each individual. As a biometric, human gait may be defined as a means of identifying individuals by the way they walk. Using gait has many advantages over other biometrics such as fingerprints most notably that it is non-invasive and available at low resolution. 
From specific distance verification, biometric systems are being flourished, ever been better in different applications and fields because of uniqueness. Biometric is derived from physiological properties of individual by its behavioral characteristics which are unique to everyone. Verification or recognition using specific method gait has become more efficient in various circumstances [4].

Every human object has different physiological properties. Purpose of the system to implement such rules, patterns or algorithms that can be used to analysis any moving body by unknown manner. In sensitive environment and in this era this is the need of circumstances that machine should be intelligent which works automatically without any manual feeding and user cooperation. Often seems sensitive environment face major issues like blur images after pixels damaged or required user cooperation [5]. So for idea of gait analysis using biometric or automatic machine is more efficient and accurate. Gait analysis covers the walking style uniquely and many subsets of walk like angles, speed, foot intensity, legs size, vein identification. This is video based technology for coming era [6]. Unique features of gait method are like changing mood, speed of moving, loading luggage or holding handbag. In this research we are using Artificial Neural Network (ANN).

Gait identification is a process of verification of any individual by unknown manner in moving state. Moving is behavior state so it is subset of biometric monitoring. Biometric monitoring enables the environment detecting the danger and threat in a while.

Gait is un-markable, so intelligence group or banks can use it without interaction and being unfriendly with people for recognition individually depending on need. Gait can be observed after all if it shows low pixels or low resolution in image. Basically "a gait is pattern of steps of an individual".
Interest in gait as a biometric has increased over the years, especially in the computer-vision arena. Niyogi and Adelson [7] and Guo et al. [8] were the first to announce gait analysis techniques capable of discriminating between individuals. The major single contributor to progress of automatic recognition by gait has been Defence Advanced Research Project's Agency (DARPA's) Human ID at a Distance research program. This program was established to improve security at United Sates embassies following some terrorists act in 1998. Mohamed Sayed (2018) states in the published work that It is crucial to find methods that analyze large amount of data captured by cameras and/or various sensors installed all around us. Machine learning becomes a prevailing tool in analyzing such data that signifies behavioral characteristics of human beings. Gait as an identifier for use in individual recognition systems has respective and almost certainly unique key features for each person including centroid, cycle length and step size. Gait is sometimes preeminent suited to recognition or surveillance scenarios. It might be used in the identification of females who are the yaring veils in some countries without critical social issues. The objective of this project is to predict accurately one-dimensional coordinates of normalized $n$-component vectors representing two dimensional silhouettes in order to identify individuals at a distance without any interaction and obtrusion. Varied algorithms are further incorporated into walk pattern analysis to adoptively improve gait recognitions and classification. The results are reported reasonable identification performance as compared to several machine learning methods [9].

Anju Susan George, Anitta Antony, Elsa Roy, Mathews Job (2017), propose an efficient selfsimilarity based gait recognition system for human identification using artificial neural networks (ANN). Initially the background modeling is done from a video sequence. Subsequently, the moving 
foreground objects in the individual image frames are segmented using the background subtraction algorithm. Then, the morphological skeleton operator is used to track the moving silhouettes of a walking figure. Finally, when a video sequence is fed, the proposed system recognizes the gait features and thereby humans, based on self-similarity measure. The proposed system is evaluated using gait databases and the experimentation on outdoor video sequences demonstrates that the proposed algorithm achieves a pleasing recognition performance [10].

Human gait has common patterns of movements and describes a rhythmic and periodic motion by which the body moves step by step in the required direction. A period of the gait cycle exists between the successive heel strikes, and the gait motion in space and time satisfies spatial and temporal symmetry. To extract the gait motion, body angles of each segment are extracted from gait silhouette images. A simplified 2D stick figure with six joint angles is used to represent the human body structure for recovering and interpreting the human movement.

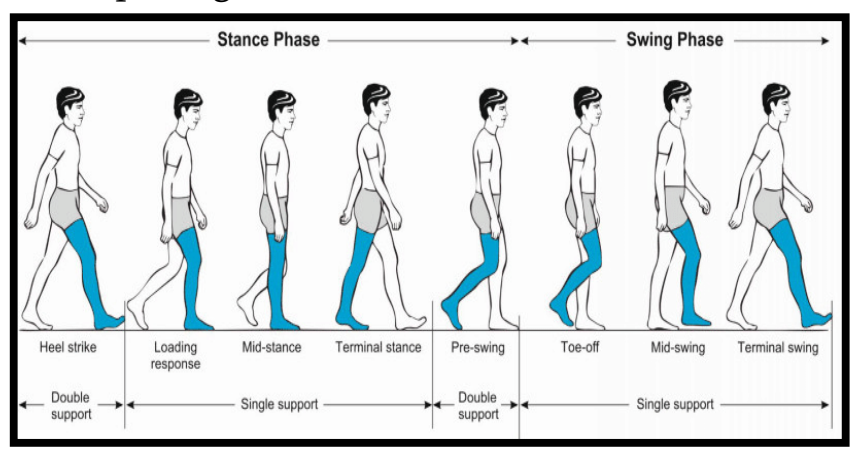

Figure 1: Normal Gait Cycle

As shown in Figure 1 human gait is described by a process of locomotion in which the moving body is supported by first one leg and then other. As the moving body passes over the supporting leg, the other leg is swung forward in preparation for its next stance phase. The time interval between two successive occurrences of one of the repetitive events of gait motion is defined as the gait cycle. Each gait cycle is separated into two distinct periods: the stance and the swing phase.

In general, the stance and swing phases have been divided into eight sub-phases: initial contact $(I C)$, loading response (lr), mid-stance (MS), terminal stance $(t s)$, pre and initial swing $(p w)$, mid-swing $(M W)$, and terminal swing $(t w)$. Each of the eight gait phases has a functional objective and a different length time interval in gait cycle.

Also, human gait motion has many modes, among which walking, running and standing are the three most often seen in daily life. These modes are the states of action in human motion, and a human can change between these states. By biomechanics definitions, standing, walking and running are distinguished firstly by the stride duration, stride length, velocities and the range of motion made by the limbs. That is, the speed of the gait motion is the most important feature to distinguish among these three states. A second difference concerns the existence of periods of double support or double float, and this is determined by the duration of the stance phase. In general, normal walking is translated to double support $(D S / I C)$ states through the single support (ss) [11]. The relationship of the states is naturally represented as a finite state machine as shown in figure below.

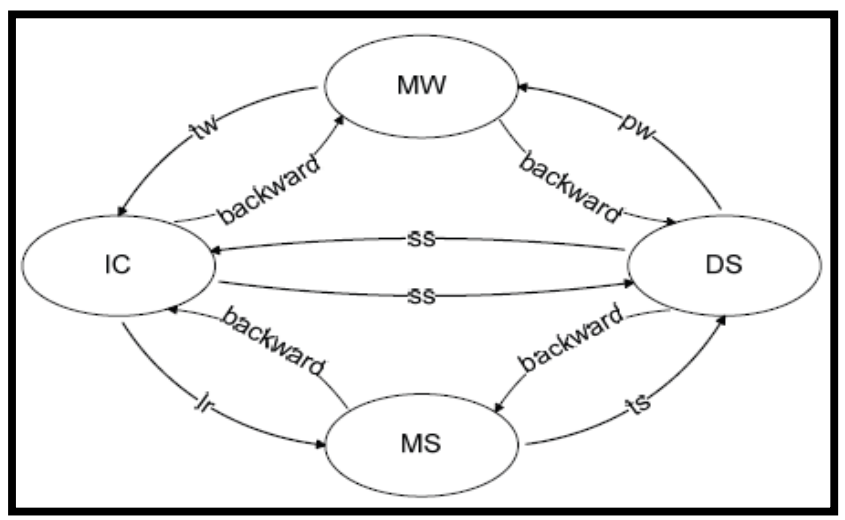

Figure 2 : Human Gait Motion Transition Diagram

The main objectives of the work done here are as under: 
1. To create an ANN based Gait Analysis for Human Identification in MATLAB.

2. To extract the gait feature vectors from the gait signatures of the Gait database.

3. To design neural network architecture for extracted feature vectors.

4. To train the ANN to retrieve the weight vectors from feature vectors.

5. To test the ANN for different input gait sequences.

6. To evaluate ANN performance on basis of recognition and speed at different training gait angles.

7. To achieve these objective the steps to be followed are Pre-processing, Feature Extraction and Classification.

\section{METHODS AND MATERIAL}

The proposed framework is illustrated in Figure 3. An identification system recognizes an individual by searching the template database for a match. However, a typical human gait identification system can be divided into training and recognition modules.

The training module is responsible for making a trained database to identify a person. During the training phase, the gait motion video is created by adding image frame sequence. A feature extractor processes this representation to generate a more compact and expressive representation such as gait feature vector. The feature vectors for each person are then trained by a pattern recognition algorithm, and the trained results will be stored in a gait identification system's database.

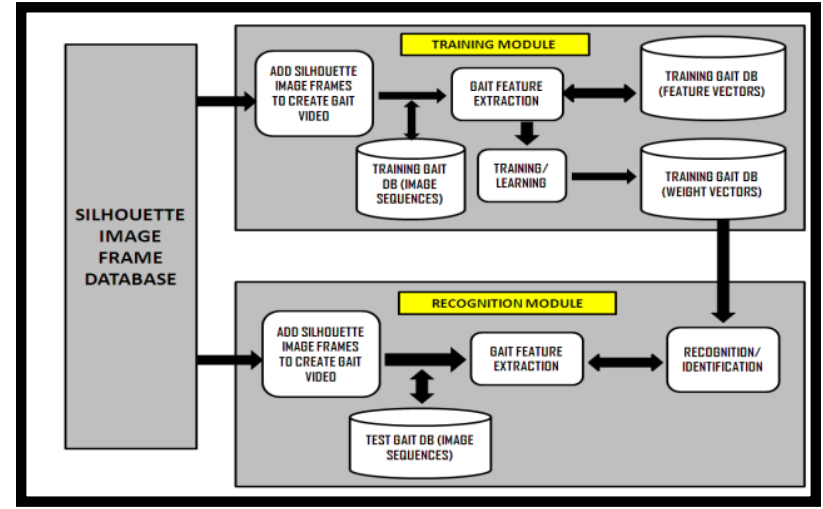

Figure 3: The Proposed Gait Recognition System

An identification system recognizes an individual by searching the template database for a match. However, a typical human gait identification system can be divided into training and recognition modules. The training module is responsible for making a trained database to identify a person. A feature extractor processes this representation to generate a more compact and expressive representation such as gait feature vector. The feature vectors for each person are then trained by a pattern recognition algorithm, and the trained results will be stored in a gait identification system's database.

In addition, the recognition module is responsible for identifying the person. During the recognition phase, the gait motion of the person to be identified is given as input which converts into the same sort of feature vector as in training. After that, the feature vector will be submitted to the recognizer, which automatically computes it against the trained database to determine the identity of the individual.

An automated pattern recognition system minimally contains an input subsystem that accepts sample pattern vectors and a decision-maker subsystem that decides the classes to which an input pattern vector belongs. If it also classifies, then it has a training phase in which it learns a set of classes of the population from a sample of pattern vectors, namely, it partitions the population into the subpopulations that are classes. As described above, a multi-layered 
feed-forward neural network is employed here to train and recognize the human gait.

Figure 4 shows the network architecture used within this study. In the figure, $\mathbf{X}$ is the input feature vector with $N$ elements, and $\mathrm{O}$ is the output vector with $M$ elements. The neural network has one hidden layer of sigmoid nodes followed by an output layer of linear nodes. To train the network, the enhanced back-propagation algorithm is used. Also, the nodes of output layer are divided into two groups, and information about a maximum output node of each group is used to decode the output to an identification code of gait.

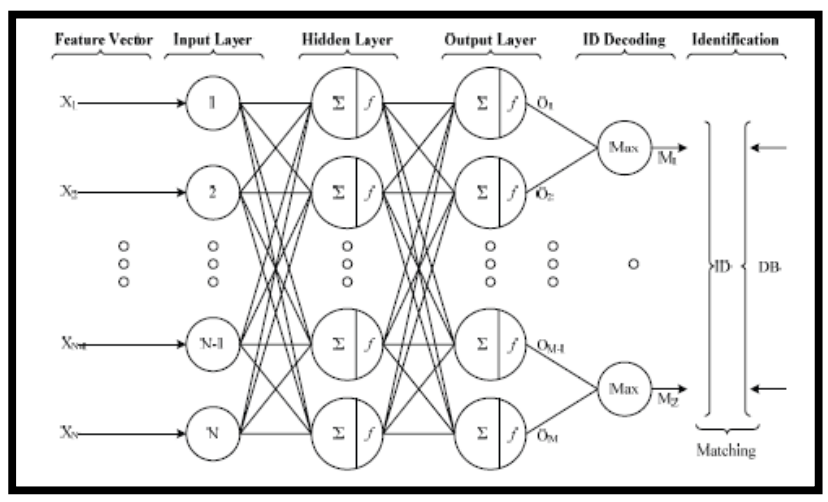

Figure 4: Two Layer Neural Network

Pre-processing is performed on the acquired images so as to optimize them for feature extraction. This process involves getting rid of redundant information, and maximizing the relevant information. In this work, pre-processing sequentially involves the steps of background subtraction, morphological operations, gait period estimation, and Frame Difference Energy Image (FDEI) reconstruction. The CASIA Gait Database readily comes with pre-processed binary silhouette. The broad outline of gait recognition process is shown in Figure 5. Gait period estimation is required for two purposes - to use gait period as a feature itself, and to separate gait cycles for further processing. Gait cycles represent the fundamental unit of human gait, and every processing attribute, for example, exemplars, clusters, ANN parameters are defined in accordance with gait cycles.

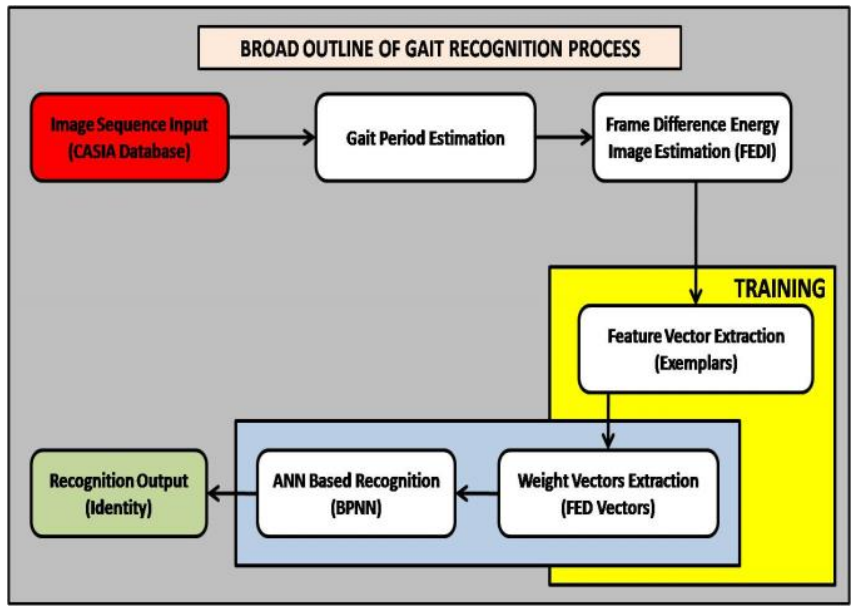

Figure 5: Broad Outline of Steps in Gait Recognition Process

But when a camera captures a moving person, it just captures a stream of digital frames. To group this stream of frames into distinct gait cycles, it is crucial to have a reliable estimate of the gait period. During any walking cycle, the following two situations are routinely encountered.

- Situation-1: When the legs of a walking subject are stretched to the maximum, i.e. when the distance between both the legs is maximum, the area under non-zero pixels is maximum.

- Situation 2: Conversely, the area under non-zero pixels is minimum when the legs cross each other.

Since walking is a quasi-periodic process, this means that the number of non-zero pixels periodically increases and decreases repeatedly as a person walks. This information is used by the described method to estimate the gait period. After the completion of background subtraction, the bottom half of each binary silhouette in the input sequence is selected and the number of non-zero (white) pixels is counted. These values are stored in a 1-D vector and plotted. The plot appears as a series of valleys and peaks, with the peaks representing Situation-1 and valleys representing Situation-2. Any one gait cycle involves two peaks and three valleys [Rest (valley) to RightFoot-Ahead (peak) to Rest (valley) to Left-FootAhead (peak) to Rest position (valley)]. An estimate 
for the gait period can be obtained by measuring the distance between the first and third valleys. Generally speaking, this can be obtained by measuring the distance (number of frames elapsed) between any two valleys (or peaks) that have one valley (or peak) between them. Figure below illustrates the plot of the vector discussed above. The peaks and valleys represent the maximum and minimum separation between limbs respectively

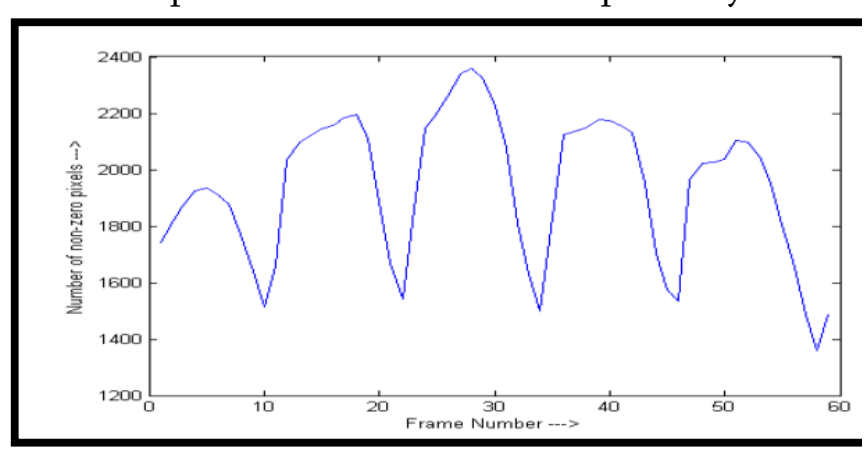

Figure 6 : Sample Plot of number of non-zero pixels in the bottom half of a silhouette over the progression of a gait cycle.

This final pre-processing step aims to make the recognition process robust to imperfections in silhouette extraction. The quality of extracted human silhouettes is directly related to and crucial for robust gait identification. Often due to exceptions and operational errors in pre-processing algorithms such as background subtraction, incomplete silhouettes are obtained. These imperfect silhouettes present a major problem, since incompleteness of silhouettes appears to be more harmful and intractable compared to other errors such as the presence of noisy artifacts, and can drastically affect recognition performance. To alleviate these effects, this work employs Frame Difference Energy Image to reconstruct the silhouettes and make the recognition process robust to imperfect silhouettes.

The primary motivation for this step is to retain the shape features of the silhouette while mitigating the detrimental effects of imperfect silhouette extraction.
The following steps outline the construction of FDEI representation of a gait cycle.

Step 1: Segment the gait cycle into $\mathrm{N}$ temporally adjacent clusters and calculate the clusteral GEI or CGEI, which is the mean of all the frames of the particular cluster. The CGEI is a broad representative of the stance of a particular cluster. This concept is the same as that of GEI which involves the same process for the entire gait cycle. Figure below illustrates the clusteral GEIs of a sample gait cycle.

Step 2: This step involves the de-noising of the clusteral GEI by means of a simple thresholding operation based on an empirically selected threshold.

Step 3: This step involves the calculation of 'positive portion' of frame difference.

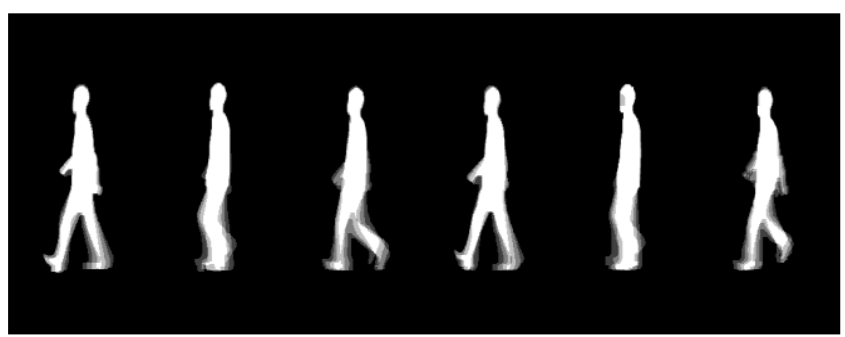

Figure 7 : Clusteral Gait Energy Images (CGEIs) of sample gait cycle

Step 4: This is the final step which involves the construction of the Frame Difference Energy Image at time $t$, denoted as $\operatorname{FDEI}(x, y, t)$, and is defined as the sum of the positive portion of frame difference $F D(x, y, t)$ as obtained in Step 3 above and the denoised CGEI $D_{c}(x, y)$ Step 2 above.

During every gait cycle, a set of certain distinct stances or positions can be identified, such as (sequentially) 1- Rest, 2- Hand raised, 3- Hands and Feet Separated, 4- Maximum Displacement between limbs, 5-Return to rest. These stances are generic in nature and each person transits through these over the gait cycle. The information contained in these stances are different for different people, both statically and temporally, and thus can be used as a 
discriminatory feature. Features corresponding to these position-points are taken as exemplars.

The motivation for using an exemplars-based method is that recognition can depend on some distance measure between the observed silhouette and the exemplars.

\section{ANN based Recognition using Feature Vector and Weight Vectors}

In this approach, $\mathrm{N}$ number of stances is picked from the gait sequence to act as exemplars, and the whole sequence and recognition process is based on this set of exemplars. This $\mathrm{N}$ also defines the number of layers on which the Artificial Neural Network (ANN) -based recognition process is based. The selection of the number of exemplars $\mathrm{N}$ is done. The primary characteristic of the indirect approach is that the higher dimensional feature vectors extracted from the binary silhouette images of the gait cycle are not directly used in the classification process. Instead, they are mapped on to or represented in a lowerdimensional space which retains most of the information relevant for classification while reducing redundancy and computational complexity.

The Frame-to-Exemplar Distance (FED) vector is a measure of reducing the higher dimensional features to lower dimension. But as the gait cycle progresses, the distance between the first exemplar and the current frame will increase, and that between the second exemplar and the current frame will decrease, till the distance becomes minimum. After that, the distance between the current frame and the second exemplar will also start increasing again, and the frame will gradually move closer to third exemplar, and so on. Thus, there will be a succession of valleys temporally corresponding to the FED vector. Most importantly, the FED vector is virtually independent of the choice of features or dimensionality of feature vectors extracted from the observed sequences.
The FED vector can be seen as the observed manifestation of the transition across exemplars or stances (a hidden stage of a neural network processing a feature vector to weight vector). The whole process can be seen as a an enhanced Back Propagation Neural Network (BPNN) algorithm for training multi-layered neural network, based on selective retraining and a dynamic adaptation of learning rate and momentum with exemplars representing the layers, and an ANN can be used to model the statistical characteristics of the process according to the observed FED vectors which are employed to recognize the gait. Thus the FED vectors represent the observation symbols of the ANN, which in turn are also called weight vectors of ANN.

The ANN parameters as well as exemplars of all the persons in the database are available, along with a probe gait sequence. The objective is to reveal the identity of the subject in the probe gait sequence by using ANN to find weight vectors that have the maximum probability of generating that particular sequence. Initialization for the parameters remains the same as in the training step while initial value for weight vector is obtained using the exemplars of each person in the gait database. For each person in the database, we already have the weight vectors parameters and exemplar vectors; BPNN is used to compute the likelihood or probability that the probe sequence was produced by the weight vector of that person. A ranking is carried out using the degree of repetition of feature vectors and weight vectors for recognition.

Figure 8 illustrates the training procedure and recognition procedure respectively in the form of flowchart. 


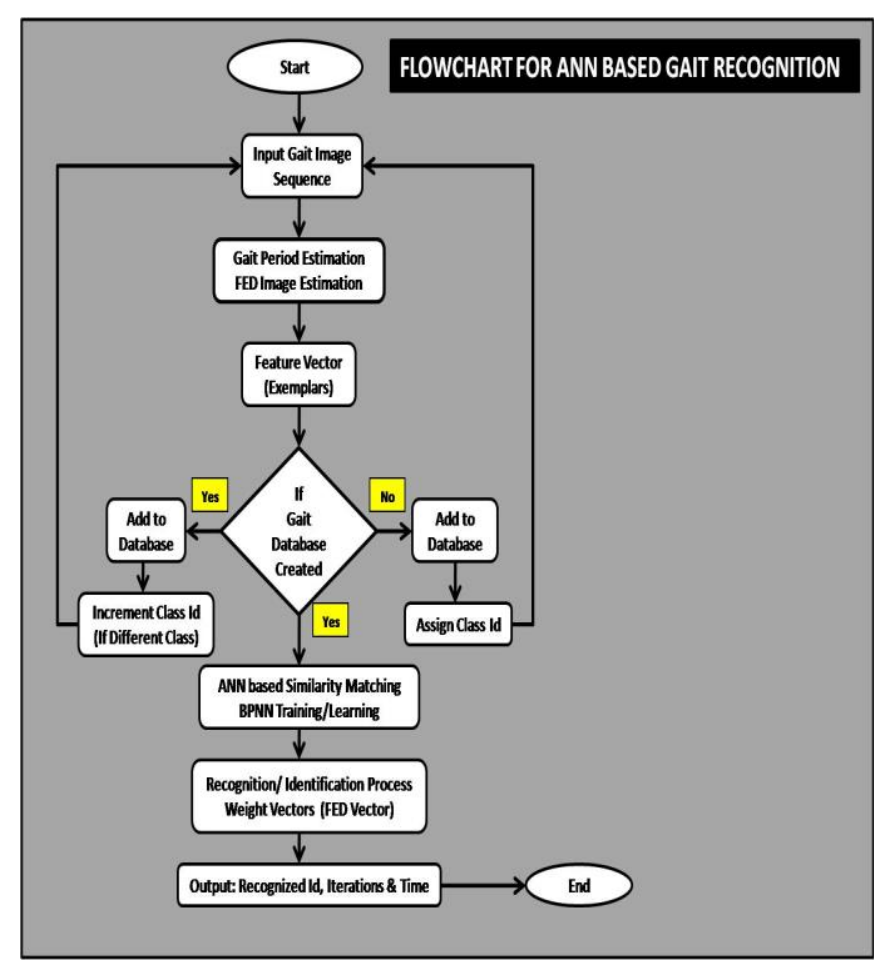

Figure 8 : Flowchart: ANN Based Gait Recognition

\section{RESULTS AND DISCUSSION}

The results are obtained for ANN based Human Gait Analysis by development of two different userinterfaces in MATLAB one Created using GUIDE and other user-interface created programmatically. The two different application GUI's for Gait Analysis using artificial neural networks are developed tested using three different database entry samples for CASIA Gait Database A which contains 20 Classes with 12 samples from each class with a total number of image sequence samples of 240 .

\section{A. The User-Interface}

The designed user interface for ANN based Human Gait Analysis using MATLAB GUIDE is shown in figure as under:

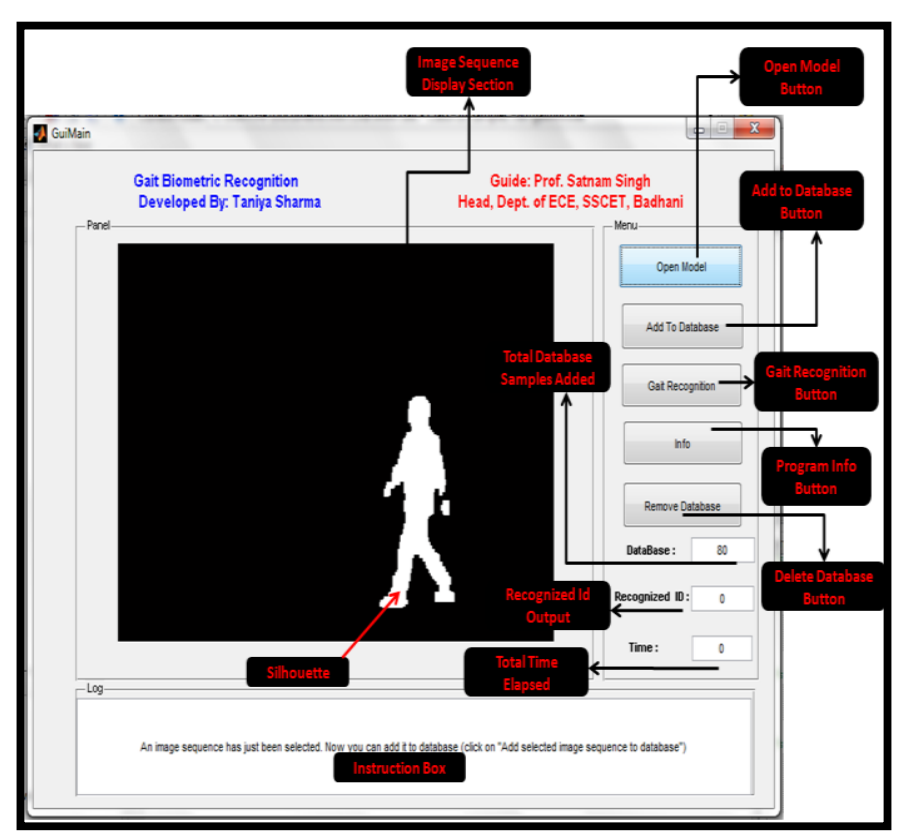

Figure 9: ANN Gait Recognition MATLAB GUI created using MATLAB GUIDE

\section{B. Performance Analysis of ANN based Gait Recognition System}

Title The ANN based Gait Recognition application is tested on three different datasets of CASIA Gait database. Each dataset consists of 20 classes where each class corresponds to an individual subject or person. The Dataset 1 also referred to as Dataset 80 consists of a total of 80 gait sequences with 4 gait sequences per class. The Dataset 2 also referred to as Dataset 160 consists of a total of 160 gait sequences with 8 gait sequences per class. The Dataset 3 also referred to as Dataset 240 consists of a total of 240 gait sequences with 12 gait sequences per class.

The testing procedure includes the testing of Dataset of gait sequences with training of ANN done at a specific gait angle of gait pattern of a particular subject. For Dataset 80 the training gait angle was selected as $0^{\circ}$. For Dataset 160 the training gait angle was selected as $45^{\circ}$. For Dataset 240 the training gait angle was selected as $90^{\circ}$. The training was done for 5000 iterations for the ANN and time elapsed for training of each dataset was recorded. 
Once required training of the ANN was done for different gait angles on different datasets, the testing phase was to be set in motion as per follows:

The ANN based Gait Recognition application for Dataset 1 also referred to as Dataset 80 consists of a total of 80 gait sequences with 4 gait sequences per class was trained for gait angle $0^{\circ}$ and training time was recorded followed by testing for all 80 input gait sequences. The results for recorded for Recognized Id and Recognition Time for each gait sequence and average recognition time for whole dataset. The training time for Dataset 80 was recorded as 2102 seconds. The average recognition time for whole dataset was recorded as 0.082615 seconds.

The ANN based Gait Recognition application for Dataset 2 also referred to as Dataset 160 consists of a total of 160 gait sequences with 8 gait sequences per class was trained for gait angle $45^{\circ}$ and training time was recorded followed by testing for all 160 input gait sequences. The results for recorded for Recognized Id and Recognition Time for each gait sequence and average recognition time for whole dataset. The training time for Dataset 160 was recorded as 4883 seconds. The average recognition time for whole dataset was recorded as 0.110965 seconds.

The ANN based Gait Recognition application for Dataset 3 also referred to as Dataset 240 consists of a total of 240 gait sequences with 12 gait sequences per class was trained for gait angle $90^{\circ}$ and training time was recorded followed by testing for all 240 input gait sequences. The results for recorded for Recognized Id and Recognition Time for each gait sequence and average recognition time for whole dataset. The training time for Dataset 240 was recorded as 7405 seconds. The average recognition time for whole dataset was recorded as 0.148341 seconds.
Finally a comparison of recorded results was done for different parameters like No. of Gait sequences per dataset, Recognized ID, Recognition Time, Average Recognition Time, Training Time and Training angle, which is shown below in different graphical representations.

\section{Analysis Graph 1: Total Gait Sequences (Dataset 80 Vs Dataset160 Vs Dataset240)}

The graph shows comparison of total no. of gait sequences in each of the three datasets. The Dataset 80 contains 80 Gait Sequences. The Dataset 160 contains 160 Gait Sequences. The Dataset 240 contains 240 Gait Sequences.

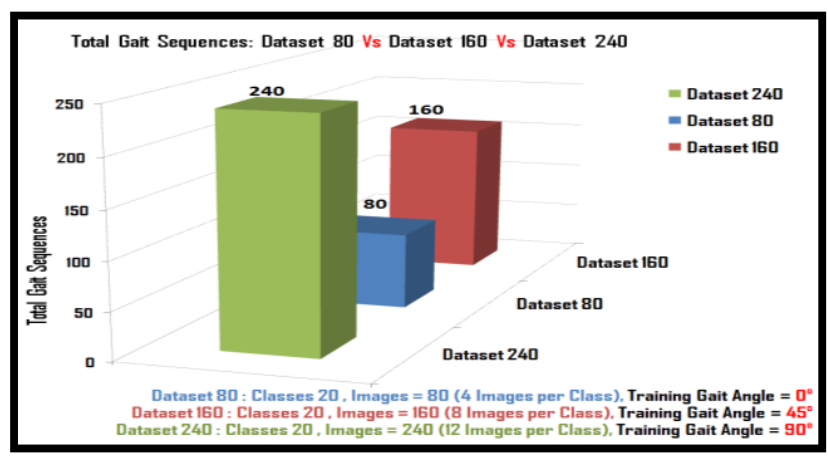

Figure 10: Total Gait Sequences in each Dataset

\section{Analysis Graph 2: ANN Training Gait Angles (Dataset} 80 Vs Dataset160 Vs Dataset240)

The graph shows comparison of training gait angles applied in each of the three datasets. The Dataset 80 is trained at $0^{\circ}$ gait angle. The Dataset 160 is trained at $45^{\circ}$ gait angle. The Dataset 240 is trained at $90^{\circ}$ gait angle.

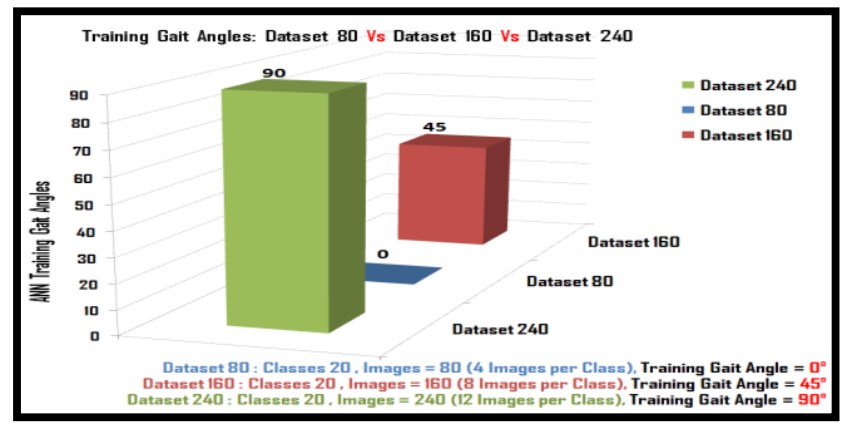

Figure 11: ANN Training Gait Angles for each Dataset 


\section{Analysis Graph 3: ANN Training Time (Dataset 80 Vs Dataset160 Vs Dataset240)}

The graph shows comparison of ANN training time in seconds for each of the three datasets at different gait angles. As the number of images for each dataset increases the training time for the dataset also increases.

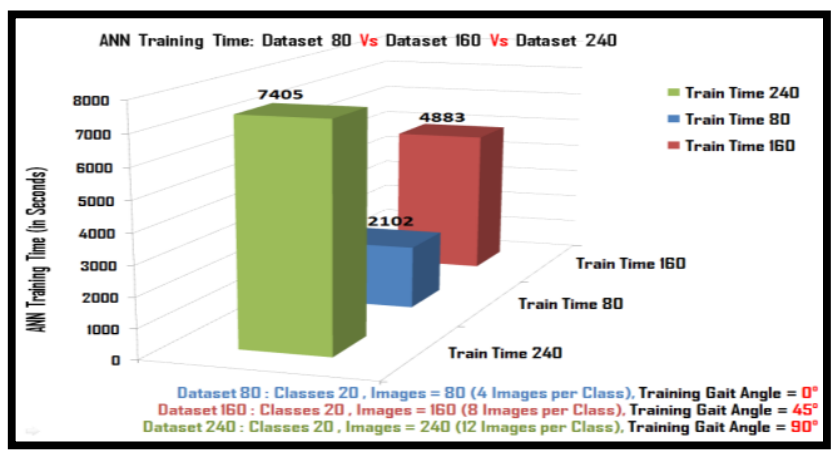

Figure 12: ANN Training Time for each Dataset

\section{Analysis Graph 4: Input Id Vs Recognized Id for Dataset 80}

The graph shows comparison of Input Gait Sequence Id and Output Recognized Id for each of the Dataset 80 for which the training was done at $0^{\circ}$ with ANN training time of 2102 seconds. The recorded results as depicted for number of classes which equals to 20 with 4 gait sequences per class amounting to a total of 80 gait sequences for the whole dataset.

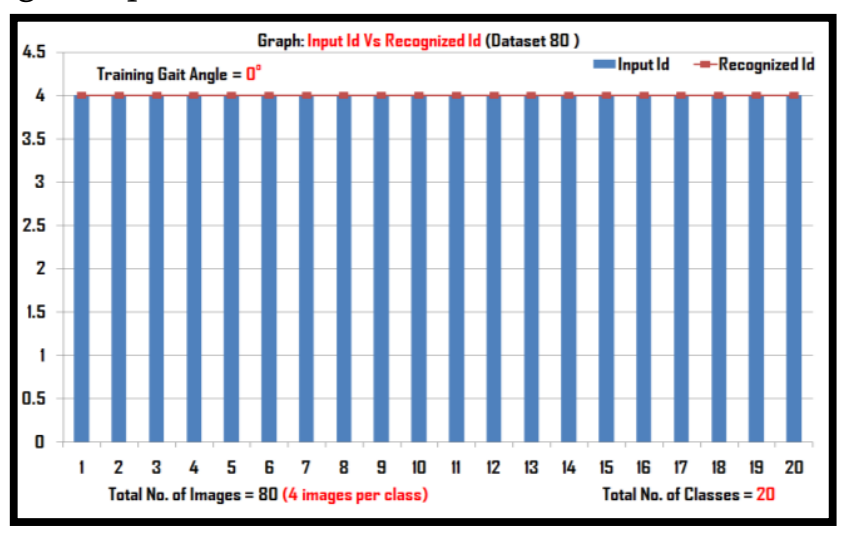

Figure 13: Input Id versus Recognized Id for Dataset 80

It is evident from the graph that for training Gait Angle of $0^{\circ}$, the ANN performs with high accuracy of $100 \%$ for the Dataset 80.
Analysis Graph 5: Input Id Vs Recognized Id for Dataset 160

The graph shows comparison of Input Gait Sequence Id and Output Recognized Id for each of the Dataset 160 for which the training was done at $45^{\circ}$ with ANN training time of 4883 seconds. The recorded results as depicted for number of classes which equals to 20 with 8 gait sequences per class amounting to a total of 160 gait sequences for the whole dataset.

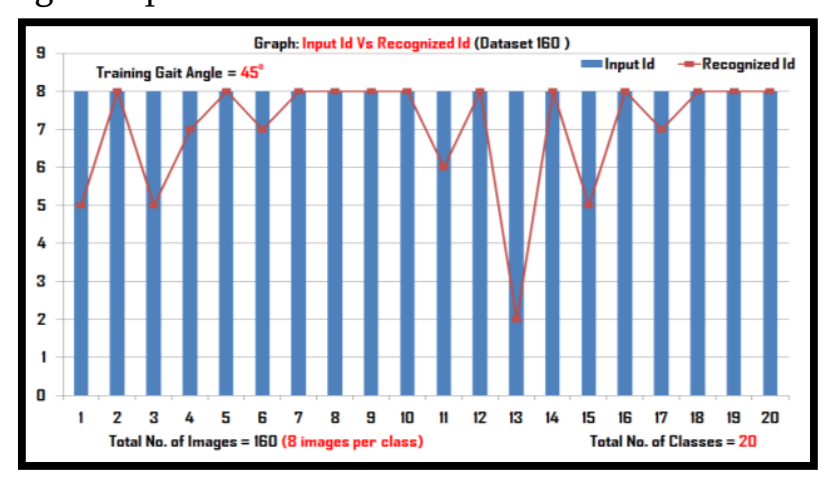

Figure 14: Input Id versus Recognized Id for

\section{Dataset 160}

It is evident from the graph that for training Gait Angle of $45^{\circ}$, the ANN performance reduces for not all but few instance inputs for classes one, three, five, six, eleven, thirteen, fifteen and seventeen but still the performance of ANN remains comprehensive for the Dataset 160.

Analysis Graph 6: Input Id Vs Recognized Id for Dataset 240

The graph shows comparison of Input Gait Sequence Id and Output Recognized Id for each of the Dataset 240 for which the training was done at $90^{\circ}$ with ANN training time of 7405 seconds. The recorded results as depicted for number of classes which equals to 20 with 12 gait sequences per class amounting to a total of 240 gait sequences for the whole dataset. 


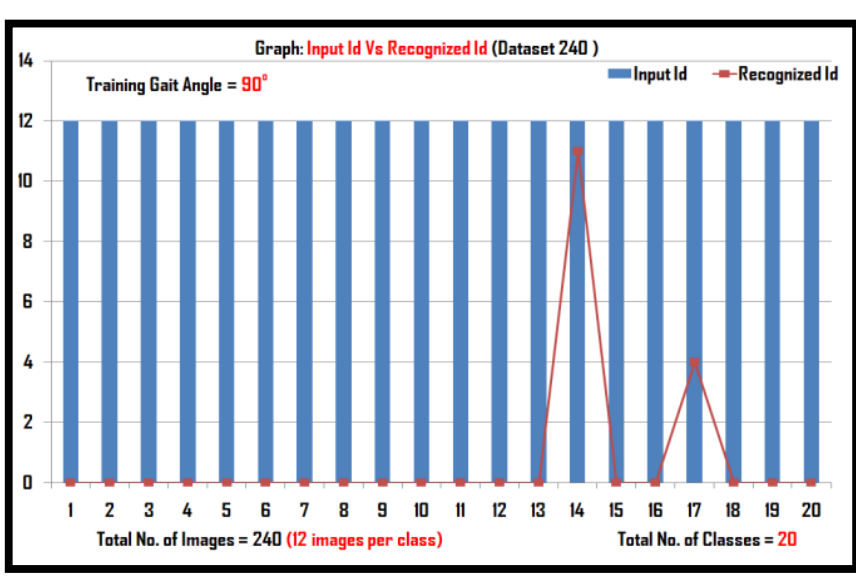

Figure 15: Input Id versus Recognized Id for Dataset 240

It is evident from the graph that for training Gait Angle of $90^{\circ}$, the ANN performance reduces completely for all instance inputs for all classes and output toggles between only two classes i.e. class fourteen and class seventeen thus giving no useful performance output for the Dataset 240.

\section{Analysis Graph 7: ANN Recognition Time (Dataset 80 Vs Dataset160 Vs Dataset240)}

The graph shows comparison of ANN Recognition time in seconds for each of the three datasets at different gait angles for all individual inputs from all 20 classes. As the size of the dataset increases the recognition time for the dataset also increases.

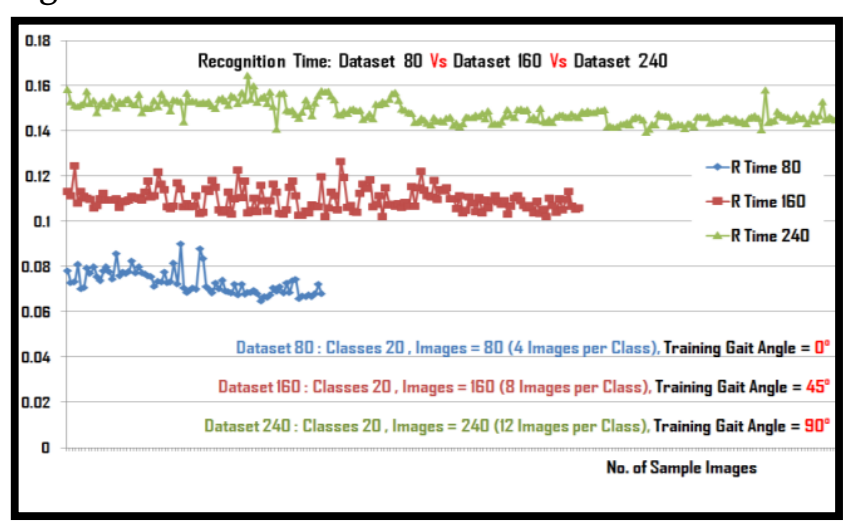

Figure 16: ANN Recognition Time Performance Analysis

The comparison show there is a minimal increase in recognition time as the size of the dataset increases but still the recognition time performance of ANN remains very high with recognition time ranging from 0.07 seconds to 0.16 seconds which is considerably low and highly rapid. This is again confirmed by another analysis of average recognition time for the whole dataset.

\section{Analysis Graph 8: ANN Average Recognition Time (Dataset 80 Vs Dataset 160 Vs Dataset 240)}

The graph shows comparison of ANN Average Recognition time in seconds for each of the three datasets at different gait angles. As the size of the dataset increases the average recognition time for the dataset also increases.

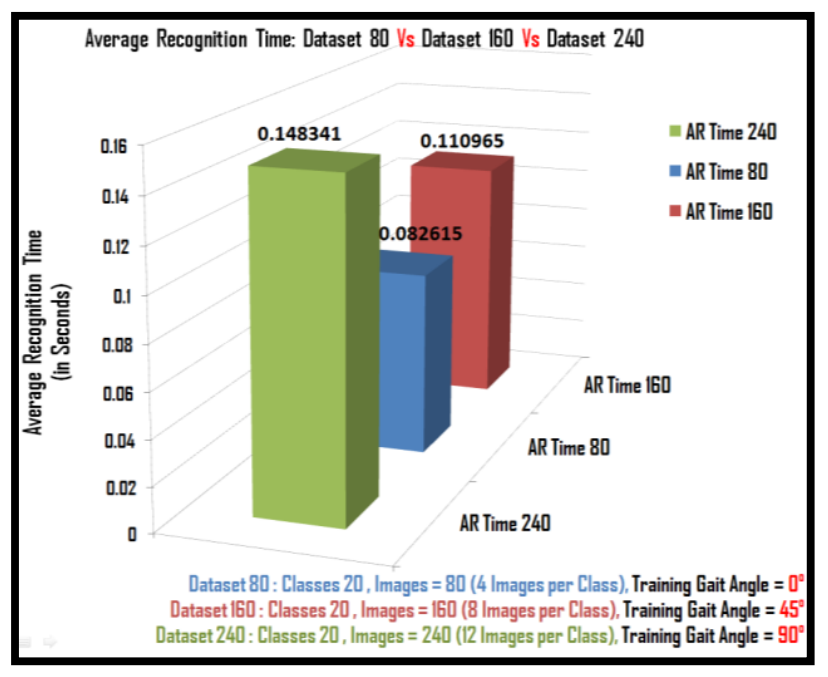

Figure 17: ANN Average Recognition Time Performance Analysis

The comparison show there is a minimal increase in recognition time as the size of the dataset increases but still the recognition time performance of ANN remains very high with average recognition time for Dataset 80 is calculated as 0.082615 seconds, for Dataset 160 the average recognition time is calculated as 0.110965 seconds and for Dataset 240 the average recognition time is calculated as 0.148341 which is considerably low and highly rapid.

\section{IV.CONCLUSION}

Although This work explores a gait recognition method with binary silhouette-based input images and Artificial Neural Network (ANN)-based 
classification. The performance of the recognition method depends significantly on the quality of the extracted binary silhouettes. A fuzzy correlogram based method is employed for background subtraction and Frame Difference Energy Image (FDEI) reconstruction is performed to make the recognition method robust to partial incompleteness of silhouettes. Feature extraction process uses extracted features directly for classification while the indirect method maps the higher-dimensional features into a lower-dimensional space by means of a Frame-to-Exemplar-Distance (FED) vector.

The FED uses the distance measure between predetermined exemplars and the feature vectors of the current frame as an identification criterion. The extracted features are fed to the ANN-based classifier which models human gait to compute similarity scores and carry out identification.

This work achieves an overall accuracy for all database datasets with difference in recognition time as the dataset sample bulk increases and the ANN trains and learns itself from increased number of samples. As expected, the results using this approach are better, owing to a basic trade-off between complexity and efficiency.

The approach is computationally more extensive with a better overall performance, while the indirect approach tends to be computationally less intensive at the cost of recognition performance. Also this approach is less vulnerable to noise and distortion compared to the indirect approach.

Surprisingly, the results obtained by using the FED vector show that in this case, the overall performance is actually slightly lesser in the case of FDEI reconstruction as compared to the use of direct binary silhouettes.
It is also seen that the recognition performance depends to a certain extent on the specific gait sequences. The algorithms used in this work produce relatively better results in the case of CASIA DatasetA.

The complete comparison and analysis of the system on recognition on basis of ANN training done at different gait angles shows that the when trained at gait angle of $0^{\circ}$, the ANN performs with $100 \%$ accuracy for Dataset 80 . When trained at gait angle $45^{\circ}$, the ANN performance starts degenerating a little and ANN gives an erroneous performance for Dataset 160. When trained at gait angle $90^{\circ}$, the ANN performance degenerates completely and ANN doesn't perform at all for Dataset 240 and toggles the output between only two classes.

On the other hand the complete comparison and analysis of ANN for recognition speed shows that the system performs with great efficiency on this front with recognition times ranging from 0.07 seconds to 0.16 seconds.

\section{REFERENCES}

[1] R. Gross and J. Shi (2001), "The cmu motion of body (mobo) database technical report," Carnegie Mellon University. CMU-RI-TR-0118, 2001.

[2] M. S. Nixon, T. Tan, and R. Chellapa, Human Identification Based On Gait. Springer, 2006.

[3] Navneet Kaur (2014), "Review On: Gait Recognition for Human Identification using NN", (IJCSIT) International Journal of Computer Science and Information Technologies, Vol. 5 (3), 2014, pp. 3991-3993

[4] [Su-li XU, Qian-jin ZHANG (2010), "Gait recognition using fuzzy principal component analysis", 2nd International Conference on ebusiness and information system security, IEEE, 27 may, 2010, pp. 23-29. 
[5] Jang-Hee Yoo and Mark S. Nixon (2011), "Automated Marker less Analysis of Human Gait Motion for Recognition and Classification”, ETRI Journal, Vol. 33, No. 2, pp 259- 266, April 2011.

[6] J.J. Abrams (2011), "Study and Analysis of Human Gait to Recognize the Person", International Journal of Science and Research (IJSR), India Online ISSN: 2319- 7064 Volume 2 Issue 5, May 2011.

[7] S. A. Niyogi and E. H. Adelson (1994), "Analyzing and recognizing walking figures in xyt," in Proceedings of the IEEE Computer Society Conference on Computer Vision and Pattern Recognition, 1994, pp. 469-474.

[8] Y. Guo, G. Xu, and S. Tsuji (1994), "Understanding human motion patterns," in In Proceedings of 12th International Conference on Pattern Recognition, 1994, pp. 325- 329.

[9] Mohamed Sayed (2018), "Biometric Gait Recognition Based on Machine Learning Algorithms", Journal of Computer Science, Vol.14, (7), pp. 1064.1073, 2018.

[10] Anju Susan George, Anitta Antony, Elsa Roy, Mathews Job (2017), “An Efficient Gait Recognition System for Human Identification using Neural Networks", International Journal of Innovations \& Advancement in Computer Science IJIACS ISSN 2347-8616 Volume 6, Issue 5, pp. 76-83, May 2017.

[11] L. Wang, W.M. Hu, and T.N. Tan (2003), "Recent Developments in Human Motion Analysis", Pattern Recognition, Vol. 36, No. 3, pp. 585-601, 2003 